\title{
Correct expression of the material derivative in continuum physics
}

Bohua Sun ${ }^{1, \text { a) }}$

School of Civil Engineering \& Institute of Mechanics and Technology, Xi'an University of Architecture and Technology, Xi'an 710055, China

(Dated: 19 April 2020)

The material derivative is important in continuum physics. This Letter shows that the expression $\frac{d}{d t}=\frac{\partial}{\partial t}+(\boldsymbol{v} \cdot \nabla)$, used in most literature and textbooks, is incorrect. The correct expression $\frac{d(:)}{d t}=\frac{\partial}{\partial t}(:)+\boldsymbol{v} \cdot[\boldsymbol{\nabla}(:)]$ is formulated.

In continuum physics, there are two ways of describing continuous media or flows, the Lagrangian description and the Eulerian description. In the Eulerian description, the material derivative with respect to time must be defined. For mass density $\boldsymbol{\rho}(\boldsymbol{x}, t)$, flow velocity $\boldsymbol{v}(\boldsymbol{x}, t)=v_{k} \boldsymbol{e}_{k}$, and stress tensor $\boldsymbol{\sigma}=\sigma_{i j} \boldsymbol{e}_{i} \otimes \boldsymbol{e}_{j}$, the material derivatives are given by:

$$
\begin{aligned}
& \frac{d \rho}{d t}=\frac{\partial \rho}{\partial t}+\frac{\partial \rho}{\partial x_{k}} \frac{\partial x_{k}}{\partial t}=\frac{\partial \rho}{\partial t}+v_{1} \frac{\partial \rho}{\partial x^{1}}+v_{2} \frac{\partial \rho}{\partial x^{2}}+v_{3} \frac{\partial \rho}{\partial x^{3}} \\
& \frac{d \boldsymbol{v}}{d t}=\frac{\partial \boldsymbol{v}}{\partial t}+\frac{\partial \boldsymbol{v}}{\partial x_{k}} \frac{\partial x_{k}}{\partial t}=\frac{\partial \boldsymbol{v}}{\partial t}+v_{1} \frac{\partial \boldsymbol{v}}{\partial x^{1}}+v_{2} \frac{\partial \boldsymbol{v}}{\partial x^{2}}+v_{3} \frac{\partial \boldsymbol{v}}{\partial x^{3}}
\end{aligned}
$$

and

$$
\frac{d \sigma}{d t}=\frac{\partial \sigma}{\partial t}+\frac{\partial \sigma}{\partial x_{k}} \frac{\partial x_{k}}{\partial t}=\frac{\partial \sigma}{\partial t}+v_{1} \frac{\partial \sigma}{\partial x^{1}}+v_{2} \frac{\partial \sigma}{\partial x^{2}}+v_{3} \frac{\partial \sigma}{\partial x^{3}},
$$

respectively, where $t$ is time, $\boldsymbol{x}=x_{k} \boldsymbol{e}_{k}$ is position, $\boldsymbol{e}_{k}$ is a base vector and $v_{k}=\frac{\partial x_{k}}{\partial t}$ is the flow velocity.

In most textbooks, handbooks and encyclopedia, such as Refs. 1-12, the above material derivatives are expressed as:

$$
\begin{aligned}
& \frac{d \rho}{d t}=\frac{\partial \rho}{\partial t}+(\boldsymbol{v} \cdot \nabla) \rho, \\
& \frac{d \boldsymbol{v}}{d t}=\frac{\partial \boldsymbol{v}}{\partial t}+(\boldsymbol{v} \cdot \nabla) \boldsymbol{v},
\end{aligned}
$$

and

$$
\frac{d \sigma}{d t}=\frac{\partial \sigma}{\partial t}+(\boldsymbol{v} \cdot \nabla) \boldsymbol{v}
$$

where the gradient operator $\nabla=e_{k} \frac{\partial}{\partial x_{k}}$.

To simplify Eqs. (1)-(3) further, a differential operator is introduced as follows:

$$
\frac{d}{d t}=\frac{\partial}{\partial t}+(v \cdot \nabla)
$$

This operator is used by most fluid mechanics textbook$\mathrm{s}$, including well-known graduate textbooks such as Landau \& Lifshitz, ${ }^{1,2}$ Prandtl, ${ }^{3,4}$ Anderson, ${ }^{5}$ Pope, ${ }^{6}$ Cengel $\&$ Cimbala, ${ }^{7}$, Kundu et al. $^{8}$, Woan ${ }^{9}$, wikipedia ${ }^{10,11}$ and britannica $^{12}$.

\footnotetext{
a)Electronic mail: sunbohua @xauat.edu.cn; http:/imt.xauat.edu.cn
}

In the latest version of Landau \& Lifshitz, ${ }^{13}$ the material derivative of flow velocity is given in another form:

$$
\frac{d v}{d t}=\frac{\partial v}{\partial t}+(v \nabla) v
$$

Besides the books mentioned above, there is a large amount of literature in fluid mechanics taking the same expressions as Refs. 1 and 13. This makes it difficult for readers to identify which expression for the material derivative is correct, causing great confusion to both students and scholars.

Although some authors, such as Lighthill, ${ }^{14}$ Batchelor, ${ }^{15}$ Frisch, ${ }^{16}$, Nhan \& Nam ${ }^{17}$, Xie, ${ }^{18}$ and Zhao ${ }^{19}$ have used the correct expression, it seems that most fluid mechanics textbooks and academic literature have adopted an incorrect expression for the material derivative as in Ref. 1. Therefore, to revive the great influence of Landau in physics and fluid mechanics, we attempt to address this issue in this dedicated paper, where we revisit the material derivative to show why Eqs. (7) and (8) are incorrect, and derive a correct expression using standard tensor calculus.

In Landau \& Lifshitz, ${ }^{1}$ the material derivative is given as:

$$
d x \frac{\partial \boldsymbol{v}}{\partial x}+d y \frac{\partial \boldsymbol{v}}{\partial y}+d z \frac{\partial \boldsymbol{v}}{\partial z}=(d \boldsymbol{r} \cdot \mathbf{g r a d}) \boldsymbol{v} .
$$

Although Landau's fluid mechanics is well known worldwide, the above expression is incorrect.

\section{Proof:}

$$
\begin{aligned}
& \text { Since } \boldsymbol{v} \cdot \operatorname{grad}=\boldsymbol{v} \cdot \boldsymbol{\nabla}=\left(v_{i} \boldsymbol{e}_{i}\right) \cdot\left(\partial_{j} \boldsymbol{e}_{j}\right)= \\
& v_{i, j} \boldsymbol{e}_{i} \cdot \boldsymbol{e}_{j}=v_{i, j} \delta_{i j}=\operatorname{div} \boldsymbol{v}, \text { and }(\boldsymbol{v} \cdot \operatorname{grad}) \boldsymbol{v}= \\
& (\boldsymbol{v} \cdot \nabla) \boldsymbol{v}=\left(v_{j, i} \boldsymbol{e}_{i} \cdot \boldsymbol{e}_{j}\right)\left(v_{k} \boldsymbol{e}_{k}\right)=\delta_{i j} v_{j, i} v_{k} \boldsymbol{e}_{k}= \\
& v_{i, i} v_{k} \boldsymbol{e}_{k}=(\operatorname{div} \boldsymbol{v}) \boldsymbol{v}, \text { where the divergence is } \\
& \operatorname{div} \boldsymbol{v}=\frac{\partial v_{1}}{\partial x^{1}}+\frac{\partial v_{2}}{\partial x^{2}}+\frac{\partial v_{3}}{\partial x^{3}}, \text { so }(\operatorname{div} \boldsymbol{v}) \boldsymbol{v}=\left(\frac{\partial v_{1}}{\partial x^{1}}+\right. \\
& \left.\frac{\partial v_{2}}{\partial x^{2}}+\frac{\partial v_{3}}{\partial x^{3}}\right) \boldsymbol{v} \text {. Thus: } \\
& \quad(\boldsymbol{v} \cdot \operatorname{grad}) \boldsymbol{v} \neq v_{1} \frac{\partial \boldsymbol{v}}{\partial x^{1}}+v_{2} \frac{\partial \boldsymbol{v}}{\partial x^{2}}+v_{3} \frac{\partial \boldsymbol{v}}{\partial x^{3}} .
\end{aligned}
$$

In the latest version of Landau \& Lifshitz, ${ }^{13}$ for reasons which are unclear, the material derivative expression has been changed to $\frac{d v}{d t}=\frac{\partial v}{\partial t}+(\boldsymbol{v} \nabla) \boldsymbol{v}$; this is also incorrect.

\section{Proof:}

Since $\boldsymbol{v} \boldsymbol{\nabla}=\left(v_{i} \boldsymbol{e}_{i}\right)\left(\partial_{j} \boldsymbol{e}_{j}\right)=v_{i, j} \boldsymbol{e}_{i} \boldsymbol{e}_{j}$,

$$
(\boldsymbol{v} \nabla) \boldsymbol{v}=\left(v_{j, i} \boldsymbol{e}_{i} \boldsymbol{e}_{j}\right)\left(v_{k} \boldsymbol{e}_{k}\right)=\left(v_{j, i} \boldsymbol{e}_{i} \boldsymbol{e}_{j}\right)\left(v_{k} \boldsymbol{e}_{k}\right),
$$


which indicates that $v \nabla$ is a 3 rd-order tensor rather than a vector. Thus:

$$
(\boldsymbol{v} \nabla) \boldsymbol{v} \neq v_{1} \frac{\partial \boldsymbol{v}}{\partial x^{1}}+v_{2} \frac{\partial \boldsymbol{v}}{\partial x^{2}}+v_{3} \frac{\partial \boldsymbol{v}}{\partial x^{3}} .
$$

To obtain the correct formulation of the material derivative, we perform some basic tensor calculus ${ }^{20}$. The del operator is a vector differential operator, and defined by $\nabla=e_{i} \frac{\partial}{\partial x_{i}}$. An important note concerning the del operator $\nabla$ is in order. Two types of gradients are used in continuum physics: left and right gradients. The left gradient is the usual gradient and the right gradient is the transpose of the forward gradient operator.

To see the difference between the left and right of gradients, consider a vector function $\boldsymbol{A}=A_{i}(\boldsymbol{x}) \boldsymbol{e}_{i}$. The left gradient of a vector $\boldsymbol{A}$ is $\boldsymbol{\nabla} \otimes \boldsymbol{A} \equiv \boldsymbol{e}_{j} \frac{\partial}{\partial x_{j}} \otimes\left(A_{i} \boldsymbol{e}_{i}\right)=\frac{\partial A_{i}}{\partial x_{j}} \boldsymbol{e}_{j} \otimes \boldsymbol{e}_{i}=A_{i, j} \boldsymbol{e}_{j} \otimes$ $\boldsymbol{e}_{i}$, or written as $\boldsymbol{\nabla} \boldsymbol{A} \equiv \boldsymbol{e}_{j} \frac{\partial}{\partial x_{j}}\left(A_{i} \boldsymbol{e}_{i}\right)=\frac{\partial A_{i}}{\partial x_{j}} \boldsymbol{e}_{j} \boldsymbol{e}_{i}=A_{i, j} \boldsymbol{e}_{j} \boldsymbol{e}_{i}$. The right gradient of a vector $\boldsymbol{A}$ is $\boldsymbol{A} \otimes \nabla \equiv\left(A_{i} e_{i}\right) \otimes e_{j} \frac{\partial}{\partial x_{j}}=$ $\frac{\partial A_{i}}{\partial x_{j}} \boldsymbol{e}_{i} \otimes \boldsymbol{e}_{j}=A_{i, j} \boldsymbol{e}_{i} \otimes \boldsymbol{e}_{j}$, or written as $\boldsymbol{A} \boldsymbol{\nabla} \equiv\left(A_{i} \boldsymbol{e}_{i}\right) \boldsymbol{e}_{j} \frac{\partial}{\partial x_{j}}=$ $\frac{\partial A_{i}}{\partial x_{j}} e_{i} e_{j}=A_{i, j} e_{i} e_{j}$, where $A_{i, j}=\frac{\partial A_{i}}{x_{j}}$.

The gradient of a scalar function is a vector, the divergence of a vector-valued function is a scalar $\boldsymbol{\nabla} \cdot \boldsymbol{A}$, and the gradient of a vector-valued function is a second-order tensor $\boldsymbol{\nabla} \boldsymbol{A}$. Although the del operator has some of the properties of a vector, it does not have them all, because it is an operator. For instance, $\boldsymbol{\nabla} \cdot \boldsymbol{A}$ is a scalar (called the divergence of $\boldsymbol{A}$ ) whereas $\boldsymbol{A} \cdot \boldsymbol{\nabla}$ is a scalar differential operator, where $\boldsymbol{A}$ is a vector. Thus the del operator $\nabla$ does not commute in this sense.

It worth to emphasise that the right gradient of a vector $\boldsymbol{A} \boldsymbol{\nabla}$ is a more natural one, is often used in defining the deformation gradient tensor, displacement gradient tensor, and velocity gradient tensor. It is clear that $\boldsymbol{A} \boldsymbol{\nabla}=(\boldsymbol{\nabla} \boldsymbol{A})^{T}$.

1. Mass density $\rho=\rho(\boldsymbol{x}, t)$ is a scalar-valued function of $\boldsymbol{x}$ and $t$ and its differential is $d \rho=\frac{\partial \rho}{\partial t} d t+\frac{\partial \rho}{\partial \boldsymbol{x}} \cdot \frac{\partial \boldsymbol{x}}{\partial t} d t$. For a scalar $\frac{\partial \rho}{\partial \boldsymbol{x}}=\rho \boldsymbol{\nabla}=\nabla \rho$, so $d \rho=\frac{\partial \rho}{\partial t} d t+(\rho \nabla) \cdot \boldsymbol{v} d t=\frac{\partial \rho}{\partial t} d t+$ $\boldsymbol{v} \cdot(\nabla \rho) d t$, or, dividing both sides by $d t$,

$$
\frac{d \rho}{d t}=\frac{\partial \rho}{\partial t}+(\rho \nabla) \cdot v=\frac{\partial \rho}{\partial t}+\boldsymbol{v} \cdot(\nabla \rho) .
$$

2. Flow velocity $\boldsymbol{v}=\boldsymbol{v}(\boldsymbol{x}, t)$ is a vector-valued function of $\boldsymbol{x}$ and $t$ and its differential is $d \boldsymbol{v}=\frac{\partial v}{\partial t} d t+\frac{\partial v}{\partial \boldsymbol{x}} \cdot \frac{\partial \boldsymbol{x}}{\partial t} d t$. For a vector $\frac{\partial \boldsymbol{v}}{\partial \boldsymbol{x}}=\boldsymbol{v} \otimes \boldsymbol{\nabla}=\boldsymbol{v} \boldsymbol{\nabla}=(\boldsymbol{\nabla} \boldsymbol{v})^{T}$. Strictly speaking, the right gradient of $\boldsymbol{v}$ should be written as $\boldsymbol{v} \otimes \nabla$; here, in order to agree as far as possible with conventional presentation, we write $\boldsymbol{v} \otimes \boldsymbol{\nabla}=\boldsymbol{v} \boldsymbol{\nabla}$ and, similarly for the left gradient, $\boldsymbol{\nabla} \otimes$ $\boldsymbol{v}=\boldsymbol{\nabla} \boldsymbol{v}$. Hence $(\boldsymbol{v} \boldsymbol{\nabla}) \cdot \boldsymbol{v}=\boldsymbol{v} \cdot(\boldsymbol{\nabla} \boldsymbol{v})$ and $d \boldsymbol{v}=\frac{\partial \boldsymbol{v}}{\partial t} d t+(\boldsymbol{v} \boldsymbol{\nabla})$. $\boldsymbol{v} d t=\frac{\partial \rho}{\partial t} d t+\boldsymbol{v} \cdot(\boldsymbol{\nabla v}) d t$, or, dividing both sides by $d t$,

$$
\frac{d \boldsymbol{v}}{d t}=\frac{\partial \boldsymbol{v}}{\partial t}+(\boldsymbol{v} \nabla) \cdot \boldsymbol{v}=\frac{\partial \boldsymbol{v}}{\partial t}+\boldsymbol{v} \cdot(\boldsymbol{\nabla} \boldsymbol{v})
$$

3. The 2nd order stress tensor $\boldsymbol{\sigma}=\boldsymbol{\sigma}(\boldsymbol{x}, t)$ is a tensorvalued function of $\boldsymbol{x}$ and $t$ and its differential is $d \sigma=\frac{\partial \sigma}{\partial t} d t+$ $\frac{\partial \sigma}{\partial \boldsymbol{x}} \cdot \frac{\partial \boldsymbol{x}}{\partial t} d t$. For an arbitrary tensor $\frac{\partial \sigma}{\partial \boldsymbol{x}}=\boldsymbol{\sigma} \boldsymbol{\nabla} \neq(\boldsymbol{\nabla} \boldsymbol{\sigma})^{T}$, hence $d \boldsymbol{\sigma}=\frac{\partial \boldsymbol{\sigma}}{\partial t} d t+(\boldsymbol{\sigma} \boldsymbol{\nabla}) \cdot \boldsymbol{v} d t$, or dividing both sides by $d t$ leads to $\frac{d \sigma}{d t}=\frac{\partial \sigma}{\partial t}+(\sigma \nabla) \cdot v$. Since $(\sigma \nabla) \cdot \boldsymbol{v}=\boldsymbol{v} \cdot(\boldsymbol{\nabla} \sigma)$,

$$
\frac{d \boldsymbol{\sigma}}{d t}=\frac{\partial \boldsymbol{\sigma}}{\partial t}+\boldsymbol{v} \cdot(\boldsymbol{\nabla} \boldsymbol{\sigma}) .
$$

If an operator must be introduced for the material derivative, it should be in the following form:

$$
\frac{d(:)}{d t}=\frac{\partial}{\partial t}(:)+\boldsymbol{v} \cdot[\nabla(:)]
$$

For a scalar function $f(\boldsymbol{x}, t)$, this leads to $\frac{d f}{d t}=\frac{\partial f}{\partial t}+\boldsymbol{v} \cdot(\nabla f)$. If $f$ is considered as a distributed function of gas molecules in their phase space, this derivative is as in Eq. (3.2) in Physical Kinetics by Lifshitz and Pitaevskii. ${ }^{2}$

For a vector function $\boldsymbol{u}(\boldsymbol{x}, t)$, Eq. (15) yields $\frac{d \boldsymbol{u}}{d t}=\frac{\partial \boldsymbol{u}}{\partial t}+$ $\boldsymbol{v} \cdot(\boldsymbol{\nabla} \boldsymbol{u})$. For a tensor function $\boldsymbol{A}(\boldsymbol{x}, t)$, Eq. (15) yields $\frac{d \boldsymbol{A}}{d t}=$ $\frac{\partial \boldsymbol{A}}{\partial t}+\boldsymbol{v} \cdot(\boldsymbol{\nabla} \boldsymbol{A})$.

Using the material derivative operator $\frac{d(:)}{d t}=\frac{\partial}{\partial t}(:)+\boldsymbol{v} \cdot[\nabla(:$ )], the Navier-Stokes momentum equation is given by $\frac{\partial v}{\partial t}+\boldsymbol{v}$. $(\boldsymbol{\nabla} \boldsymbol{v})=v \nabla^{2} \boldsymbol{v}-\frac{1}{\rho} \boldsymbol{\nabla} p$, where $p$ is flow pressure and $v$ is kinematic viscosity.

Assuming det $\boldsymbol{\nabla} \boldsymbol{v} \neq 0$, the Navier-Stokes momentum equation can be rewritten as:

$$
\begin{aligned}
\boldsymbol{v} & =\left(v \nabla^{2} \boldsymbol{v}-\frac{1}{\rho} \boldsymbol{\nabla} p-\frac{\partial \boldsymbol{v}}{\partial t}\right) \cdot(\boldsymbol{\nabla v})^{-1} \\
& =(\boldsymbol{v} \boldsymbol{\nabla})^{-1} \cdot\left(v \boldsymbol{\nabla}^{2} \boldsymbol{v}-\frac{1}{\rho} \boldsymbol{\nabla} p-\frac{\partial \boldsymbol{v}}{\partial t}\right) .
\end{aligned}
$$

If the incorrect material derivative $\frac{d}{d t}=\frac{\partial}{\partial t}+(\boldsymbol{v} \cdot \boldsymbol{\nabla})$ is used in the Navier-Stokes momentum equation, then Eq. (16) cannot be obtained.

The mistake in the definition $\frac{d}{d t}=\frac{\partial}{\partial t}+(\boldsymbol{v} \cdot \boldsymbol{\nabla})$ is mainly the incorrect representation of $\boldsymbol{v} \cdot \boldsymbol{\nabla} ; \boldsymbol{\nabla}$ is a gradient operator rather than a vector, so that:

$$
\begin{aligned}
& \boldsymbol{v} \cdot \boldsymbol{\nabla}=\left(v_{1} \boldsymbol{e}_{1}+v_{2} \boldsymbol{e}_{2}+v_{3} \boldsymbol{e}_{3}\right) \cdot\left(\boldsymbol{e}_{1} \frac{\partial}{\partial x^{1}}+\boldsymbol{e}_{2} \frac{\partial}{\partial x^{2}}+\boldsymbol{e}_{3} \frac{\partial}{\partial x^{3}}\right) \\
& =\frac{\partial v_{1}}{\partial x^{1}}+\frac{\partial v_{2}}{\partial x^{2}}+\frac{\partial v_{3}}{\partial x^{3}} \neq v_{1} \frac{\partial}{\partial x^{1}}+v_{2} \frac{\partial}{\partial x^{2}}+v_{3} \frac{\partial}{\partial x^{3}} .
\end{aligned}
$$

In conclusion, the material derivative operator is correctly defined as: $\frac{d(:)}{d t}=\frac{\partial}{\partial t}(:)+\boldsymbol{v} \cdot[\boldsymbol{\nabla}(:)]$.

Availability of data: There are no data in this theoretical article.

${ }^{1}$ L. D. Landau and E. M. Lifshitz, Fluid Mechanics (English version) (1987).

${ }^{2}$ E. M. Lifshitz and L. P. Pitaevskii, Physical Kinetics (Elsevier (Singapore) Ltd, 2008)

${ }^{3}$ L. Prandtl, Führer durch die Strömungslehre, Herbert Oertel (ed.) (Springer Vieweg, 2012).

${ }^{4}$ L. Prandtl, Prandtl's essentials of fluid mechanics, Herbert Oertel (ed.) (Springer-Verlag, 2004).

5 J. D. Anderson, Computational Fluid Dynamics-the basics with applications (The McGraw-Hill Companies, Inc., 1995).

${ }^{6}$ S. B. Pope, Turbulent flows (Cambridge University Press, 2000).

${ }^{7}$ Y. A. Cengel and J. M. Cimbala, Fluid Mechanics: Fundamentals and Applications (McGraw-Hill Education, Inc., 2010). 
${ }^{8}$ P. K. Kundu, I. M. Cohen and D. R. Dowling, Fluid Mechanics (Elsevier, 2012).

${ }^{9}$ G. Woan, The Cambridge Handbook of Physics Formulas, Cambridge University Press (2000)

${ }^{10} \mathrm{https} / / /$ en.wikipedia.org/wiki/Gauge_covariant_derivative

${ }^{11}$ https://en.wikipedia.org/wiki/Navier_Stokes_equations

${ }^{12} \mathrm{https} / / / \mathrm{www}$.britannica.com/science/Navier-Stokes-equation

${ }^{13}$ L. D. Landau and E. M. Lifshitz, Fluid Mechanics (Russian version) (2017).

${ }^{14}$ J. Lighthill, Waves in Fluids (Cambridge University Press, 1978)
${ }^{15}$ G. K. Batchelor, An introduction to fluid dynamics (Cambridge University Press, 2007)

${ }^{16}$ U. Frisch, Turbulence (Cambridge University Press, 1995)

${ }^{17}$ P.T. Nhan and M.D. Nam, Understanding viscoelasticity an introduction to rheology (Springer-Verlag Berlin Heidelberg,2013).

${ }^{18}$ X. C. Xie, Modern Tensor Analysis and Its Applications in Continuum Mechanics (Fudan University Press, 2014)

${ }^{19}$ Y. P. Zhao, Lectures on Mechanics (Science Press, China, 2018)

${ }^{20}$ J.N. Reddy, An Introduction to Continuum Mechanics, (Cambridge University Press NY, 2007). 